\title{
The measurement of emissivity of charring ablative materials
}

by. COLOMBO G., GALFETTI L. and SALERNO A.

Dipartimento di Energetica, Politecnico di Milano, Piazza L. Da Vinci, 32

20133 Milano, Italy.

\begin{abstract}
Normal emissivity of ablative thermal insulators at different temperatures is determined comparing the radiation emitted and the surface temperature for a cylindrical sample. A microthermocouple (diameter $50 \mu \mathrm{m}$ ) is carefully inserted under a very thin sublayer of the surface sample and a $\mathrm{CO}_{2}$ laser is used to heat it at different temperatures. The sample is observed by an infrared camera which allows the determination of the radiating energy emitted from its surface in the wavelength interval of $3.0-5.4 \mu \mathrm{m}$.
\end{abstract}

\section{Nomenclature}

$\begin{array}{ll}\text { A } & \text { surface area } \\ c_{O} & \text { speed of light in vacuum } \\ h & \text { Planck's constant } \\ \text { L } & \text { radiance } \\ T & \text { absolute temperature }\end{array}$

Greek Symbols

$\varepsilon \quad$ emissivity

$\theta$ co-latitude in spherical coordinate system

$\kappa \quad$ Boltzmann's constant

$\lambda$ wavelength

$\varphi$ azimuthal angle in spherical coordinate system

$\tau$ transmittance

$\omega \quad$ solid angle

$\phi \quad$ radiant flux

\section{Subscripts \\ b blackbody \\ w window}

\section{Introduction}

Experimental research activities in the field of aerospace propulsion typically concern reacting materials such as solid propellants and ablative thermal insulators.

Standard instruments and investigation methods are often not applicable and new techniques have to be devised to measure the wanted properties [1] [2].

Emissivity of ablative materials, used as thermal protections for space vehicles, is an important parameter to characterize their behavior and consequently to optimize their use.

Previous works [3] underline the importance of measuring emissivity during the ablation process, due to the strong dependence of this property on the material surface properties: the heating process required to measure emissivity affects both, density and porous structure of the pyrolized material, thus stressi

perform the measurement during the material degradation. 


\section{Experimental set up}

The experimental set up (Fig. 1) is composed of several parts.

The test chamber contains the sample holder and is endowed with two infrared windows and one optical window.

A nitrogen circulation is created in the chamber to prevent the sample from burning in air when high temperatures are reached.

The $\mathrm{CO}_{2}$ laser produces a beam of radiating energy at a wavelength of $10.6 \mu \mathrm{m}$, and it is set to different power levels to heat the sample at different iemperatures.

The infrared camera is an AVIO TVS 2700TE whose detector is sensible in the range between 3.0 and $5.4 \mu \mathrm{m}$, thus avoiding the possibility of errors due to the reflection or scattering of the laser beam.

A cylindrical sample $(15 \mathrm{~mm}$ in diameter and $10 \mathrm{~mm}$ in height) is positioned in the test chamber.

A microthermocouple (Pt/Pt-Rh 10\%,50 $\mu \mathrm{m}$ ) is inserted in a sublayer of the sample surface and must be in good thermal contact with the surface.

A digital oscilloscope is used to detect the signal of the microthermocouple.

The positioning of the microthermocouple just few microns under the surface of the sample is critical for the success of the technique.

The consistency of the sample is similar to that of a strong rubber.

The microthermocouple is inserted with a thin needle and after this operation the sample is carefully inspected, to see if the thermocouple was inserted in too much depth: in this case the sample is rejected.

This inspection is done both: optically, with the use of a microscope and thermographically, using a very low current to heat the thermocouple and seeing in the infrared how visible the heated thermocouple is under the sample surface.

The thermocouple is also disposed in a $Z$ shape to allow the determination in the infrared of the exact position of the thermocouple junction, being the two corners of $Z$ equally distant from the junction (Fig. 2).

\section{Theoretical considerations}

The spectral radiance $\mathrm{L}_{\lambda}$, em is the radiant flux emitted at the wavelength $\lambda$, in the direction $(\theta, \varphi)$ per unit of emitting surface normal to this direction, per unit solid angle at this direction, and per unit wavelength interval $d \lambda$ at $\lambda$.

$$
L_{\lambda, \mathrm{em}}(\lambda ; \theta, \varphi)=\frac{\mathrm{d} \phi_{\mathrm{em}}}{\mathrm{dA} \cdot \cos \theta \cdot \mathrm{d} \omega \cdot \mathrm{d} \lambda} .
$$

For a blackbody, the spectral radiance is a function of the temperature and the wavelength following the Planck law:

$$
L_{\lambda, b}(\lambda, T)=\frac{2 h c_{0}^{2}}{\lambda^{5} \cdot\left[\exp \left(h c_{0} / \lambda k T\right)-1\right]}
$$

The spectral directional emissivity is defined as:

$$
\varepsilon_{\lambda ; \theta}(\lambda ; \theta, \varphi ; T)=\frac{L_{\lambda, e m}(\lambda ; \theta, \varphi ; T)}{L \lambda, b(\lambda, T)}
$$

In our case, if $T_{2}$ is the surface temperature measured by the thermocouple and $T_{1}$ is the radiant temperature measured from the thermocamera, having set the emissivity to 1 , 
http://dx.doi.org/10.21611/qirt.1994.018

the radiation impinging on the sensor is equal to that emitted from a blackbody at temperature $T_{1}$ in the wavelength interval between 3.0 and $5.4 \mu \mathrm{m}$.

$$
\mathrm{L}_{\mathrm{b}}\left(\lambda_{1} \rightarrow \lambda_{2}, \mathrm{~T}_{1}\right)=\int_{\lambda_{1}}^{\lambda_{2}} \mathrm{~L}_{\lambda, \mathrm{b}}\left(\lambda, \mathrm{T}_{1}\right) \mathrm{d} \lambda=\int_{\lambda_{1}}^{\lambda_{2}} \varepsilon(\lambda, 0) \cdot \mathrm{L}_{\lambda, \mathrm{b}}\left(\lambda, \mathrm{T}_{2}\right) \mathrm{d} \lambda
$$

Assuming the emissivity to be constant with $\lambda$ (gray body) and calling $\varepsilon_{\mathbf{n}}$ the normal emissivity:

$$
\varepsilon_{\mathrm{n}}=\frac{\int_{L_{\lambda, b}}^{\lambda_{2}}{ }_{\lambda_{1}}^{\lambda_{L}}{ }_{\lambda, b}\left(\lambda, T_{2}\right) \mathrm{d} \lambda}{\lambda_{1} \lambda}
$$

The gray body assumption is important; otherwise the different sensibility of the sensor to different wavelengths should be considered.

In case that between the sample and the infrared camera there is an infrared window with transmittance $\tau_{\mathbf{w}}$, the equation becomes:

$$
\int_{\lambda_{1}}^{\lambda_{2}} L_{\lambda, b}\left(\lambda, T_{1}^{\prime}\right) d \lambda=\int_{\lambda_{1}}^{\lambda_{2}} \varepsilon(\lambda, 0) \cdot \tau_{w}(\lambda, 0) \cdot L_{\lambda, b}\left(\lambda, T_{2}\right) d \lambda
$$

where $T_{1}$ is the new temperature read by the infrared camera.

\section{Test procedure}

After the sample has been placed on the axis of the laser beam and after the exact position of the thermocouple junction has been identified by the infrared camera, the thermocouple is connected with a digital oscilloscope and the laser is switched on.

The temperature of the irradiated surface increases until it reaches a steady value.

The transmissivity of the infrared window in front of the infrared camera was calibrated in advance using a commercial blackbody.

In base to a conversion table obtained from this calibration, the thermocouple temperature $T_{2}$ was converted to a temperature $T_{3}$ which was the temperature that the infrared camera should have read, after the absorption from the infrared window, if the emissivity had been set at the correct value.

The emissivity value was then obtained, adjusting the emissivity on the infrared camera, so that the temperature read in correspondence to the thermocouple junction was T3.

In order to measure the emissivity at different temperatures, the power of the laser beam has to be changed and the whole procedure repeated.

Some problems can arise if the material cracks during the experiment in the position of the thermocouple joint, thus preventing the thermocouple from being in good thermal contact with the material and possibly exposing the thermocouple junction directly to laser radiation.

Another problem is due to the gaussian shape of the laser beam and the fact that if the thermocouple moves (because of thermal dilatation for instance), the point, at which the 
http://dx.doi.org/10.21611/qirt.1994.018

infrared camera reads the temperature, may not coincide with the thermocouple joint which can be at a different temperature.

\section{Results}

Figures 4, 5 and 6 show the results obtained from experiments made on three different kinds of materials that are conventionally named: $A B L 1, A B L 2$ and $A B L 3$.

It is possible to see that the scattering of data is proportional to the temperature at which the emissivity is evaluated.

It is known [4] that, for strongly absorbing materials, the emissivity exclusively depends on the conditions at the surface of the material.

The intrinsic difficulty of the experiment is due to the fact that the surface is reacting during the measurement.

Cracks can form on the surface and produce misleading measurements from the thermocouple that can be directly irradiated from the laser.

The ablation process can produce gases responsible for the absorption of the radiation omitted by the surface.

For $\mathrm{ABL} 1$, results have a good reproducibility up to $300^{\circ} \mathrm{C}$. Between $300^{\circ} \mathrm{C}$ and $500^{\circ} \mathrm{C}$ there is an increasing dispersion of data. Above $500^{\circ} \mathrm{C}$ the dispersion of data increases too much to reach any conclusion for emissivity evaluation.

For $A B L 2$ the dispersion of data begins at $175^{\circ} \mathrm{C}$. The higher dispersion of data for this material can be considered as a consequence of the larger cracking of the surface, compared to the others. Another explanation can also be found in the larger production of absorbing gases during the ablation process.

The dispersion of data for ABL3 is comparable to that of $A B L 1$.

The results obtained are good for temperatures under $300^{\circ} \mathrm{C}$, satisfactory for temperatures up to $500^{\circ} \mathrm{C}$ and not significant above $500^{\circ} \mathrm{C}$.

The agreement with previous works [3] is good.

The value for the hemispherical emissivity [5] can be obtained, with reasonable approximation, from the normal emissivity through a multiplying factor equal to 0,935 [6].

\section{Conclusions}

This procedure has shown to be very interesting for evaluating the normal emissivity of reacting materials at different temperatures.

The results, for the range of temperature under $500^{\circ} \mathrm{C}$, are in good agreement with the few results that can be found in the literature.

Several sources of errors can be sighted out as the cause of the excessive data dispersion for high temperatures.

These sources of errors can be minimized, but not eliminated for these materials.

Particular attention must be paid to the correct insertion of the thermocouple under the sample surface.

Microcracks of the heated surface are an important source of errors, preventing the correct evaluation of emissivity at high temperatures.

\section{REFERENCES}

[1] BALAGEAS (D.) - 'Contrôle non destructif des matérieax composites par thermographie infrarouge'. 'La Revue des Laboratoires d'essais', n.35, mai 1993. ONERA, N.1993-74.

[2] BALAGEAS (D.) - 'Les possibilités de la thermographie infrarouge pour le contrôle des matériaux composites'. Journées thematiques DRET sur 'Les matériaux composites structureax', Paris, 4-5 mars 1993. Onera N. 1993-28. 
http://dx.doi.org/10.21611/qirt.1994.018

[3] KATZOFF (S.), editor - 'Symposium on Thermal Radiation of Solids'. NASA SP-55, 1965.Paper $n .27$ by Wilson, R. G., 'Hemispherical Spectral Emittance of Ablation Chars, Carbon and Zirconia (to 3700K)', pp. 259-275.

[4] ECKERT (E. R. G.), DRAKE (R. M. Jr.) - 'Analysis of Heat and Mass Transfer'. Mc.GrawHill, 1972.

[5] BREWSTER (M. Q.) - 'Thermal Radiative Transfer and Properties'. Wiley, 1992.

[6] MAGLIC (K. D.), CEZAIRLIYAN (A.), PELETSKY (V. E.), editors - 'Compendium of Thermophysical Property Measurement Methods'. Vol. 1, Plenum Press, 1984.

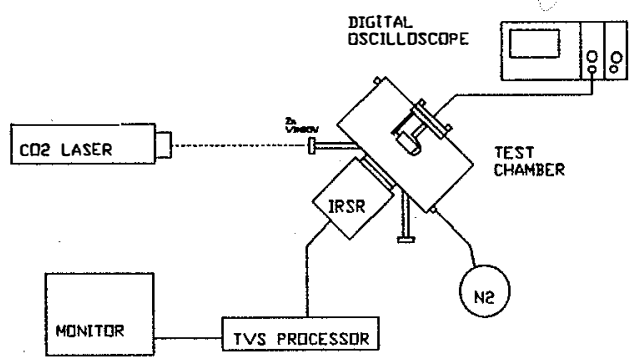

Fig.1. - Experimental set up.

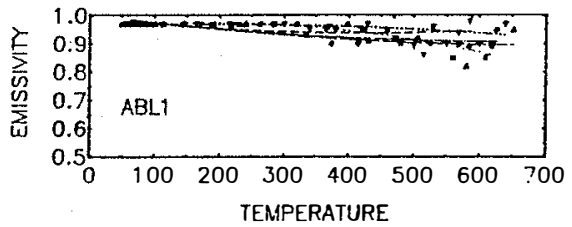

Fig. 4. - Emissivity vs temperature for $A B L 1$.

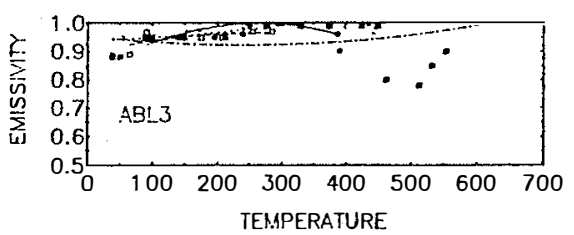

Fig. 6. - Emissivity vs temperature for ABL3.

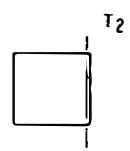

sormple with

tierinocouple

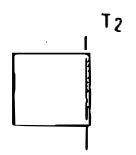

somple witl thermocouple
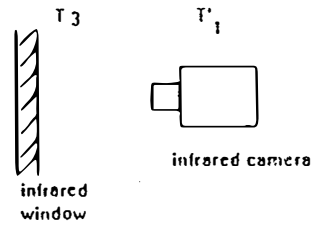

Fig. 3. - Different temperatures read by the infrared camera due to the presence of window.

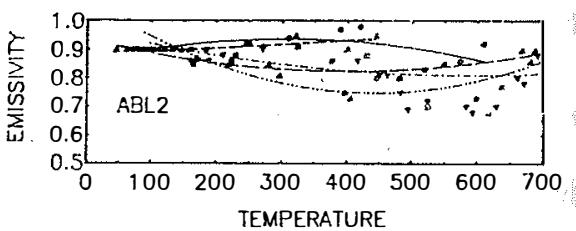

Fig. 5. - Emissivity vs temperature for ABL2. 